\title{
Evaluación de la degradación, consumo voluntario y comportamiento productivo de ovinos alimentados con saccharina y maíz
}

\section{Evaluation of degradation, voluntary consumption and productive behavior of sheep fed with saccharin and corn}

\author{
Beatriz Godínez-Juárez ${ }^{1}$, Luis M. Vargas-Villamil ${ }^{1}$, Roberto González-Garduño ${ }^{2 *}$, Juan M. Zaldívar-Cruz ${ }^{1}$, \\ Francisco Izquierdo-R ${ }^{1}$, Omar Hernández-Mendo ${ }^{3}$, Jesús A. Ramos-Juárez ${ }^{1}$ \\ ${ }^{1}$ Colegio de Postgraduados, Campus Tabasco. Periférico Carlos A. Molina, Km 3.5. Carretera Cárdenas-Huimanguillo. CP. \\ 86500. H. Cárdenas, Tabasco, México. \\ ${ }^{2}$ Universidad Autónoma Chapingo. Unidad Regional Universitaria Sursureste, Km. 7 Carretera Teapa-Vicente Guerrero, \\ Ranchería San José Puyacatengo, CP. 86800. Teapa, Tabasco, México. \\ ${ }^{3}$ Colegio de Postgraduados, Campus Montecillo. Carretera México-Texcoco Km. 36.5, Montecillo, Texcoco, CP. 56230 . Estado \\ de México, México. \\ ${ }^{*}$ Autor de correspondencia: robgarduQhotmail.com
}

Artículo científico recibido: 29 de agosto de 2016 aceptado: 10 de febrero de 2017

RESUMEN. El objetivo del estudio fue evaluar el consumo voluntario y el comportamiento productivo de ovinos alimentados con una dieta de Saccharina con maíz molido (MM), así como la degradación ruminal in situ en bovinos. Se analizó el consumo voluntario del alimento y la ganancia de peso en 24 ovinos Katahdin x Pelibuey en crecimiento, con un peso inicial promedio de $17 \pm 3.0 \mathrm{~kg}$, alimentados con cuatro dietas $(10,20,30$ y $40 \%$ de MM). En dos bovinos canulados se evaluó la degradación efectiva (DE) de la materia seca (MS) y de la fibra detergente neutro (FDN) a las $0,2,4,6,12,24,36 \mathrm{~h}$ como factor A y cinco niveles de MM (0, 10, 20, 30 y $40 \%)$ como factor B, para lo cual se utilizó una distribución factorial en un diseño completamente al azar. El tratamiento con $10 \%$ de MM presentó los valores más altos de degradación in situ, tanto para la MS como para la FDN, el cual estuvo relacionado con un alto nivel de fracción soluble y una baja fracción no degradable. Las fracciones no degradables, las tasas de degradación, y la degradación efectiva, fueron similares $(\mathrm{p} \geq 0.05)$. La degradación efectiva aumentó a partir del tratamiento con $10 \%$ de MM. Los ovinos alimentados con $40 \%$ de MM tuvieron el mayor consumo $(70.7 \mathrm{~kg}$ ) y la mayor ganancia de peso $(0.124 \mathrm{~kg})$. La aportación de maíz molido a la dieta, mejoró la degradación in situ en bovinos, el consumo y las ganancias de peso de ovinos.

Palabras clave: Alimentación, crecimiento de corderos, degradación efectiva, fermentación sólida, ganancia de peso

ABSTRACT. The objective of the study was evaluate 1 the voluntary consumption and the productive behavior of sheep fed with a diet of Saccharin with ground corn (GC), as well as ruminal degradation in situ in bovines. Voluntary feed consumption and weight gain were analyzed in 24 growing Katahdin $x$ Pelibuey sheep, with an initial average weight of $17 \pm 3.0 \mathrm{~kg}$, fed with four diets $(10,20,30$ and $40 \%$ of GC). The effective degradation (ED) of dry matter (DM) and neutral detergent fiber (NDF) was evaluated in two cannulated bovines at $0,2,4,6,12,24,36$ hours as factor A and five levels of GC $(0,10,20,30$ and $40 \%)$ as factor B, for which factorial distribution was used in a completely randomized design. Treatment with $10 \%$ of GC presented the highest values of in situ degradation for both DM and NDF, which was related to a high level of soluble fraction and a low non-degradable fraction. Non-degradable fractions, degradation rates, and effective degradation were similar $(p \geq 0.05)$. Effective degradation increased from 10 $\%$ GC treatment. The bovines fed with $40 \%$ of GC had the highest consumption during $(70.7 \mathrm{~kg})$ and the highest gain of weight $(0.124 \mathrm{~kg})$. The contribution of ground corn to the diet improved in situ degradation in bovines, consumption and weight gains of bovines.

Key words: feeding, lamb growth, effective degradation, solid fermentation, weight gain 


\section{INTRODUCCIÓN}

En la ganadería extensiva, la principal limitante en la producción animal es la disponibilidad y calidad del forraje (GonzálezGarduño et al. 2013), debido a que la dieta de los rumiantes está basada en gramíneas, cuya productividad y calidad depende de factores ambientales como la temperatura, precipitación, radiación solar, tipo de suelo, entre otros (Mohamed et al. 2004). En los climas cálidos del trópico se tienen diversos forrajes que aportan alto contenido de energía, entre los que destaca la caña de azúcar (Saccharum officinarum); gramínea con rendimientos de forraje de entre 60 y $120 \mathrm{t} \mathrm{ha}^{-1}$ año (Aguilar-Rivera et al. 2011) y de 7 a $14 \mathrm{t} \mathrm{ha}^{-1}$ de azúcar, la cual puede ser una alternativa para alimentación de ruminantes (Missio 2016). El uso de la caña molida tiene limitantes nutricionales debido a su bajo contenido de proteína cruda ( 2 al $3 \%$ ) y poco contenido mineral (Aguirre et al. 2010); también tiene lenta tasa de pasaje (Martín 2009) y baja digestibilidad de fibra (Oliveira et al. 2012), por lo que como alimento único no cubre los requerimientos de los rumiantes (NRC 2007). Para mejorar su calidad nutritiva se ha optado por aplicarle la fermentación en estado sólido (FES), en la que se desarrollan microorganismos en materiales sólidohúmedos naturales o sintéticos, que actúan sólo como soporte, impregnados en la solución que contiene la sustancia nutritiva (Julián-Ricardo y RamosSánchez 2007). En la caña, la FES se realiza por su alto contenido de azúcares que se usan como fuente de energía para los microorganismos (Ramos et al. 2006, Martín 2009). La Saccharina es un producto obtenido por fermentación de los tallos de caña de azúcar desprovistos de las hojas, suplementado con urea y sales minerales, que se somete al proceso de FES (Elías et al. 1990). Para mejorar la calidad nutritiva de la Saccharina, se ha optado por añadir algunos productos durante el proceso de fermentación, como los granos de maíz (Elías y Lezcano 1994) o sorgo (Blardony-Ricardez et al. 2013), además de desechos de cítricos, pulido de arroz y otros ingredientes energéticos o proteicos; para disminuir el contenido de fibra e incrementar la eficiencia de fermentación (Ramos et al. 2006, Cárdenas et al. 2008).

La evaluación de la degradación efectiva (DE), permite medir la cantidad de material disponible para el animal después de la degradación del alimento; con esta técnica, se puede comparar la eficiencia con la que el alimento consumido es aprovechado en el rumen del animal (Noguera y Posada 2007). Lo anterior ayuda a comprender el comportamiento productivo del animal (SalinasChavira et al. 2013). Por ello, el objetivo del presente trabajo fue estudiar el efecto de la inclusión de maíz molido en dietas integrales basadas en caña de azúcar fermentada en sólido (Saccharina), en el cambio de la degradación efectiva in situ en bovinos, y en el consumo y cambio de peso de ovinos de pelo.

\section{MATERIALES Y MÉTODOS}

\section{Localización}

El estudio de crecimiento de los corderos se realizó en una unidad de producción de ovinos en Pueblo Nuevo, Municipio de Salto de Agua, Chiapas, el cual se encuentra a una altitud de $85 \mathrm{msnm}$, con coordenadas $17^{\circ} 34^{\prime} \mathrm{LN}$ y $92^{\circ} 20^{\prime} \mathrm{LO}$. El clima de la región es Af $(\mathrm{m}) \mathrm{w}^{\prime \prime}\left(\mathrm{i}^{\prime \prime}\right) \mathrm{g}$, es decir, cálido húmedo con lluvias todo el año. La temperatura promedio anual es $26.6{ }^{\circ} \mathrm{C}$ y la precipitación de 3 289 mm (García 1988).

\section{Elaboración de las dietas}

Para las dietas se utilizó caña de azúcar (54 a $84 \%$ ), pasta de soya $4 \%$, maíz molido en proporciones de 10, 20, 30 y $40 \%$ (MM), urea $1 \%$, sulfato de magnesio $0.5 \%$ y minerales $0.5 \%$ (mezcla comercial de macro y micro-minerales para ovinos). La caña de azúcar se obtuvo en la misma unidad de producción donde se realizó el experimento, la cual se cortó y se eliminaron las hojas un día antes de su utilización, para luego moler los tallos en una picadora de forrajes marca Power de 8 $\mathrm{HP}$, modelo PFA 3000. Para después mezclar todos los ingredientes y extender la mezcla sobre piso de cemento y dejar fermentar por $24 \mathrm{~h}$. Para luego em- 
Tabla 1. Análisis bromatológico de la Saccharina adicionada con diferentes porcentajes de maíz molido.

\begin{tabular}{ccccc}
\hline $\begin{array}{c}\text { Porcentaje de } \\
\text { maíz }\end{array}$ & $\begin{array}{c}\text { Humedad } \\
(\%)\end{array}$ & $\begin{array}{c}\text { Materia Seca } \\
(\%)\end{array}$ & $\begin{array}{c}\text { Proteína cruda } \\
(\%)\end{array}$ & $\begin{array}{c}\text { Fibra detergente } \\
\text { neutro }(\%)\end{array}$ \\
\hline 0 & 68.63 & 31.37 & 12.50 & 47.05 \\
10 & 67.53 & 32.47 & 15.75 & 40.60 \\
20 & 64.93 & 35.07 & 14.01 & 36.13 \\
30 & 61.58 & 38.42 & 15.11 & 32.04 \\
40 & 53.77 & 46.33 & 14.00 & 22.32 \\
\hline
\end{tabular}

paquetarla para su conservación en bolsas de polietileno de $15 \mathrm{~kg}$, a las que se les extrajo el aire para permitir la fermentación anaerobia (Monroy et al. 2006). Los períodos de conservación del alimento fueron de ocho a $12 \mathrm{~d}$, el alimento se suministró a los animales de acuerdo a las cantidades diarias establecidas en el experimento. De cada una de las dietas se tomó una muestra y se determinó la composición química y la fracción de FDN (Tabla 1) de acuerdo con AOAC (Horwitz y Latimer 2005) y Van Soest et al. (1991).

\section{Comportamiento productivo de ovinos}

Para evaluar el comportamiento productivo se utilizaron 24 corderos cruza Katahdin $\times$ Pelibuey, destetados, de cuatro meses de edad, con peso promedio de $17 \pm 3.0 \mathrm{~kg}$, con los que se formaron cuatro grupos de seis coderos. El experimento tuvo una duración de $77 \mathrm{~d}$, sin contar el período de adaptación, por lo que en total fueron $92 \mathrm{~d}$. En este período se midió y registró el consumo de alimento y el peso vivo (PV). Actividad que se realizó por la mañana antes de ofrecer el alimento del día. La ganancia diaria de peso se determinó cada semana para obtener el cambio de peso vivo entre los días transcurridos.

Los corderos se colocaron en corrales individuales con piso de cemento, cada corral contaba con comedero y bebedero individual. Los corderos se desparasitaron al inicio del experimento con albendazol a dosis de $10 \mathrm{mg} \mathrm{kg}{ }^{-1} \mathrm{PV}$ por vía oral, además se realizó un muestreo de sangre para determinar el volumen celular aglomerado y descartar problemas de anemia por parásitos. También se vacunaron contra septicemia hemorrágica (Pasteurella multocida) y fiebre carbonosa (Clostridium spp). El periodo de adaptación fue de dos semanas, proporcionando Saccharina en la primera semana, y una combinación de Saccharina con MM de acuerdo a los porcentajes establecidos en cada tratamiento durante la segunda semana. El alimento se proporcionó dos veces al día, proporcionando de 2 a 3 kg para permitir un rechazo de al menos $30 \%$, y así asegurar que el consumo fuera a libertad. Para medir el consumo voluntario de cada uno de los animales, se registró el peso del alimento ofrecido y el rechazado de forma individual. La eficiencia de ganancia de peso se calculó como la ganancia de peso (diferencia entre el peso final - peso inicial) de cada animal, entre el consumo total de cada animal ( $\mathrm{kg}$ de ganancia de peso vivo / $\mathrm{kg}$ de MS consumida).

\section{Degradación in situ en bovinos}

Se determinó el contenido de MS y FDN de la Saccharina, Saccharina $+10 \%$ de MM, Saccharina $+20 \%$ de MM, Saccharina + $30 \%$ de MM y Saccharina $+40 \%$ de MM. La degradación se obtuvo de tres muestras de cada uno de los tratamientos, las cuales se introdujeron en dos bovinos machos (Orskov y McDonald 1979), fistulados, cruza de Holstein $\times$ Cebú, de cuatro años de edad, para obtener un total de seis repeticiones por muestra, con excepción de las muestras del tiempo cero, con tres repeticiones. Se evaluaron siete tiempos $(0$, $2,4,6,12,24$ y $36 \mathrm{~h}$ ), por lo que en total se tuvieron 195 muestras. Después de la degradación ruminal las bolsas se extrajeron y se lavaron con agua corriente, para después secarlas en estufa y pesarlas. Posteriormente se calculó la degradación con la fórmula: Porcentaje de degradación $=($ peso inicial de la muestra - peso residual de la muestra) / 
(peso inicial de la muestra) X 100. Con los valores obtenidos del porcentaje de degradación se estimó la degradación efectiva a las $36 \mathrm{~h}$.

\section{Degradación efectiva y variables cinéticas}

Para determinar la DE de la MS (DEMS), fracción no degradable (U) de la MS (UMS), fracción soluble (FS) de la MS (FSMS), tasa de degradación (kd) de la MS (kdMS), DE de la FDN (DEFDN), U de la FDN (UFDN), FS de la FDN (FSFDN) y kd de la FDN (kdFDN) a las $1000 \mathrm{~h}$, se modificó un sistema de ecuaciones diferenciales desarrollado por Olivera et al. (2014) a partir de los conceptos de degradación efectiva (Noguera y Posada 2007). La modificación permitió estimar las variables indicadas, y con un sistema de cuatro ecuaciones diferenciales, se describió el comportamiento de variables de estado, S, D, SE y DE, con el siguiente sistema de ecuaciones: $\mathrm{dSdt}=$-Degradación, $\mathrm{dSEdt}=$ Degradacion_efectiva-Pasaje, dDdt=Degradación, $\mathrm{dDEdt}=$ Degradación_efectiva, $\quad$ Pasaje $=\mathrm{kp} * \mathrm{SE}$, Degradación $=k d^{*} S$, Degradación_efectiva $=k^{*}$ SE y US $=U+S$. Con valores iniciales: $S_{0}=100-$ FS-U; $\mathrm{SE}_{0}=100-\mathrm{FS}-\mathrm{U} ; \mathrm{D}_{0}=\mathrm{FS} ; \mathrm{DE}_{0}=\mathrm{FS} ; \mathrm{U}=10$; $F S=50 ; k d=0.03 ; k p=0.03$. Dónde: $S=$ Cantidad de substrato residual utilizado para la descripción de la $\mathrm{D}, \mathrm{SE}=$ Cantidad de substrato residual utilizado para la descripción de la $\mathrm{DE}, \mathrm{U}=$ Material no degradable, $\mathrm{D}=$ Material degradado, $\mathrm{DE}=$ Material degradado tomando en cuenta la tasa de pasaje y $\mathrm{FS}=$ Fracción soluble. La variable $\mathrm{D}$ se ajustó a las curvas de degradación in situ de cada tratamiento para estimar la U, FS y kd. La DE correspondió al valor de la variable del mismo nombre, utilizando un $\mathrm{kp}=0.03$, a las $1000 \mathrm{~h}$. Para el ajuste de los parámetros y la obtención de la DE se utilizó el paquete de computo Berkeley Madonna v8.01.

\section{Diseño experimental y análisis estadístico}

Debido a que en una prueba preliminar se observó que una dieta a $0 \% \mathrm{MM}$, generaba pérdidas de peso en ovinos, se descartó este tratamiento. Pero se consideró en las pruebas de degradación, con el fin de discriminar las características nutricionales de la Saccharina en relación con las dietas donde se incluyó el MM. Para la evaluación de la degradación se realizó un experimento de factorial $5 \times 7$; donde el factor A fue la dieta (Saccharina + porcentaje de $M M$ ), que se evaluó en los niveles de $0,10,20$, 30 , y $40 \%$ de MM; y el factor B fue el tiempo de degradación a las $0,2,4,6,12,24$, y 36 h. La combinación de los dos factores y sus respectivos niveles generó un total de 35 tratamientos, que se alojaron en un diseño experimental de bloques al azar generalizado con tres repeticiones. Cada uno de los dos bovinos fistulados utilizados en el experimento constituyó un bloque. Para evaluar la degradación de la MS, y la degradación de la FDN, se realizó un análisis de varianza de acuerdo al diseño experimental propuesto y al siguiente modelo lineal mixto: $\mathrm{Y}_{i j k l}=\mu+\beta_{i}+\alpha_{j}+\gamma_{k}+\pi_{i j k}+\theta_{j k}+\mathrm{e}_{i j k l}$; Donde: $\mathrm{i}$ $=1,2 ; \mathrm{j}=1,2, \ldots, 5 ; \mathrm{k}=1,2, \ldots, 7 ; \mathrm{I}=1,2,3$.

Donde: $Y_{i j k l}$, representa la observación en el i-ésimo animal o bloque, j-ésimo nivel del factor $A$ con el k-ésimo nivel del factor $\mathrm{B}$ y la l-ésima repetición; $\mu$, es la media general; $\beta_{i}$, es igual al efecto aleatorio del i-ésimo animal; $\alpha_{j}$, equivale al efecto fijo del j-ésimo nivel del factor $A ; \gamma_{k}$, es el efecto fijo del k-ésimo nivel del factor $\mathrm{B} ; \pi_{i j k}$, es el efecto aleatorio de la interacción animal $\times \mathrm{A} \times \mathrm{B} ; \theta_{j k}$, es la interacción $\mathrm{A} \times \mathrm{B}$ y $\mathrm{e}_{i j k l}$ es el error aleatorio. De igual forma, la DE, la FS y la kd fueron analizadas de acuerdo al diseño experimental mencionado, pero sin incluir el factor tiempo de degradación (horas) en el modelo: $\mathrm{Y}_{i j k l}=\mu+\beta_{i}+\alpha_{j}+\mathrm{e}_{i j k l}$.

Para analizar las variables peso vivo inicial, peso vivo final, consumo de MS, ganancia diaria de peso (GDP), y eficiencia de la ganancia de peso, se utilizó un diseño experimental completamente al azar con seis repeticiones. Los tratamientos evaluados fueron las dietas con 10, 20, 30 y 40 $\%$ de MM. Para la interacción $A \times B$ (Dieta por tiempo) y efectos principales que fueron significativos ( $p \leq 0.05)$ se realizó la prueba de comparación múltiple de medias de Tukey. También se efectuaron contrastes ortogonales entre los tratamientos de inclusión de MM 10 y 20 contra 30 y $40 \%$ para las variables consumo de MS, ganancia diaria de peso (GDP), y eficiencia de la ganancia de peso. La in- 
Tabla 2. Efecto de la interacción dieta por tiempo en la degradación de la materia seca y la fibra detergente neutro.

\begin{tabular}{lllllllll}
\hline \multirow{2}{*}{ Variable } & \multirow{2}{*}{ Dieta* } & \multicolumn{7}{c}{ Tiempos de degradación (h) } \\
\cline { 3 - 8 } & & 0 & 2 & 4 & 6 & 12 & 24 & 36 \\
\hline \multirow{4}{*}{ MS } & 0 & $57.18^{b}$ & $59.06^{c}$ & $64.02^{b}$ & $69.44^{b c}$ & $73.21^{b}$ & $75.43^{b}$ & $75.57^{b}$ \\
& 10 & $65.67^{a}$ & $72.44^{a}$ & $73.27^{a}$ & $76.30^{a}$ & $80.49^{a}$ & $81.17^{a}$ & $82.45^{a}$ \\
& 20 & $56.73^{b}$ & $69.98^{b}$ & $68.40^{b}$ & $73.78^{b a}$ & $76.79^{b a}$ & $81.10^{a}$ & $84.07^{a}$ \\
& 30 & $59.61^{b}$ & $59.84^{c}$ & $64.38^{b}$ & $69.17^{c}$ & $75.81^{b}$ & $81.48^{a}$ & $83.93^{a}$ \\
& 40 & $47.20^{c}$ & $61.18^{c}$ & $64.11^{b}$ & $70.47^{b c}$ & $75.77^{b}$ & $82.93^{a}$ & $86.29^{a}$ \\
\hline \multirow{4}{*}{ FDN } & E.E & 1.52 & & & & & & \\
& 0 & $17.38^{b}$ & $19.20^{c}$ & $29.08 \mathrm{~d}$ & $40.93^{b c}$ & $48.01^{b}$ & $48.92^{b a}$ & $53.73^{b a}$ \\
& 10 & $45.10^{a}$ & $48.05^{a}$ & $52.82^{a}$ & $54.76^{a}$ & $58.61^{a}$ & $56.19^{a}$ & $60.37^{a}$ \\
& 20 & $39.41^{a}$ & $42.27^{b a}$ & $48.93^{b a}$ & $50.28^{b a}$ & $49.16^{b}$ & $53.49^{b a}$ & $61.52^{a}$ \\
& 30 & $47.91^{a}$ & $36.23^{b}$ & $42.93^{b c}$ & $42.58^{b c}$ & $47.45^{b}$ & $46.45^{b c}$ & $54.42^{b a}$ \\
& 40 & $22.50^{b}$ & $43.41^{b a}$ & $36.47^{d c}$ & $38.57^{c}$ & $31.91^{c}$ & $38.48^{c}$ & $46.52^{b}$ \\
\cline { 2 - 8 } & E.E & 3.27 & & & & & & \\
\hline
\end{tabular}

$a b c$ Medias con diferente superíndice en la misma columna son estadísticamente diferentes $(\mathrm{P} \leq 0.05)$. * Saccharina con el porcentaje respectivo de maíz molido. E.E Error estándar de la media.

formación se procesó con los procedimientos GLM y MIXED del software para análisis estadístico SAS versión 9.2 .

\section{RESULTADOS}

\section{Degradación efectiva y variables cinéticas}

En la FES se observaron diferencias en las variables DMS y DFDN que se pueden atribuir a la interacción dieta y tiempo de degradación $(p<$ $0.01)$ y al efecto aleatorio del animal $(p<0.05)$. La interacción entre las dietas y los tiempos, indica que cada dieta tuvo diferente comportamiento en cada tiempo de observación (Tabla 2). La degradación de la MS de la dieta sin MM (0\%) presentó los valores más bajos en todos los tiempos evaluados después de las $0 \mathrm{~h}(57.18 \%)$, mientras que en el tiempo 0 , la dieta con $40 \%$ evidenció el valor más bajo (47.2 $\%)$ pero finalizó con el valor más alto $(86.3 \%)$ a las $36 \mathrm{~h}$ (Tabla 2). Mientras que la dieta con $10 \%$ de MM presentó los valores más altos de degradación en comparación con las otras dietas hasta las $12 \mathrm{~h}$. Pero a las 24 y $36 \mathrm{~h}$ todas las dietas fueron similares, con excepción de la dieta con $0 \%$ de MM (Tabla 2, Figura 1). Para los promedios de las dietas, se observó que el tratamiento con $10 \%$ de MM tuvo los promedios de degradación de MS (75.97\%) y de FDN (53.7 \%) más altos, y se comportaron de manera diferente $(p<0.05)$ que las otras dietas
(Tabla 3). Para la FDN, las dietas con 10 y $20 \%$ de MM tuvieron los mayores valores de degradación, mientras que las dietas con 0 y $40 \%$ de MM tuvieron los menores valores en casi todos los tiempos.

El análisis de varianza presentó diferencias significativas $(p \leq 0.05)$ entre las dietas para las variables DEMS, FSMS, kdMS, DEFDN, FSFDN y kdFDN (Tabla 4). Para la DEMS el menor valor lo tuvo la dieta con $0 \%$ de MM (73.60\%), con diferencias significativas $(p<0.05)$ con las otras dietas, mientras que entre las dietas de 10 a $40 \%$ no se observaron diferencias. La UMS al $0 \%$ tuvo el valor más alto con respecto a las otras dietas, encontrándose únicamente diferencia estadística ( $\mathrm{p}<$ 0.05 ) con la dieta al $40 \%$ de MM. La FSMS fue superior en las dietas con 10 y $20 \%$ de MM ( $<<0.05)$. En la kdMS no se observaron diferencias entre las dietas (Tabla 4). La mayor DEFDN correspondió a las dietas de 10 a $20 \%$ de MM, mientras que las dietas de 0,30 y $40 \%$ presentaron los valores más bajos sin diferencias estadísticas entre ellas. Para la UFDN se tuvo el mayor valor en la dieta sin inclusión de MM (47.54 \%) y el más bajo con $40 \%$ de inclusión $(p<0.01)$. La FSFDN tuvo el valor más bajo con la dieta al $0 \%$ de MM (18.7\%). Las dietas con 10, 20, y $30 \%$ de MM fueron las que presentaron los valores más altos de FSFDN, sin diferencias estadísticas entre ellas, pero si entre las dietas con 10 y $40 \%$ de MM. El parámetro kdFDN 




no presentó diferencias estadísticas, pero los valores se redujeron a partir del $10 \%$ de MM (Tabla 4).

Tabla 3. Efecto de la dieta en la degradación de la materia seca y fibra detergente neutro.

\begin{tabular}{|c|c|c|}
\hline \multirow{2}{*}{ Dieta } & \multicolumn{2}{|c|}{ Degradación (\%) } \\
\hline & MS & FDN \\
\hline Saccharina & $67.70^{d}$ & $36.75^{d}$ \\
\hline Saccharina $+10 \%$ maíz & $75.97^{a}$ & $53.70^{a}$ \\
\hline Saccharina $+20 \%$ maíz & $72.55^{b}$ & $49.29^{b}$ \\
\hline Saccharina $+30 \%$ maíz & $70.60^{c}$ & $45.42^{c}$ \\
\hline Saccharina $+40 \%$ maíz & $69.71^{d}$ & $36.84^{d}$ \\
\hline Desviación estándar & 0.41 & 0.87 \\
\hline
\end{tabular}

\section{Comportamiento productivo de ovinos}

En la engorda de los corderos, la dieta con 40 $\%$ de MM mostró el consumo de MS más alto durante el periodo de estudio (70.69 kg MS), seguida de la dieta con $30 \%$ (59.60 kg MS), con diferencias significativas con las otras dietas $(p<0.01)$, pero no se encontraron diferencias en el consumo entre las dietas con 10 y $20 \%$ de MM (Tabla 5). Los contrastes ortogonales mostraron que las dietas con 10 y 20 vs 30 y $40 \%$ de inclusión fueron diferentes estadísticamente (Tabla 5).

\section{DISCUSIÓN}

La dieta de Saccharina con $10 \%$ de MM tuvo la mayor degradación (Tabla 3). Las diferencias observadas de los otros tratamientos con relación al 0 $\%$ se pueden atribuir al incremento en la cantidad de azúcares aportados por el MM en la dieta, debido al incremento en la fracción soluble, y la disminución de la fracción no degradable, en la MS y la FDN (Tabla 4), así como la aportación de energía, que fue aprovechada por los microorganismos (Ramos et al. 2006). Lo que confirma la importancia de considerar en los estudios de degradación, el tiempo de inclusión de un control ( $0 \%$ de MM) en la evaluación de dietas fermentadas, ya que existen diferencias en la degradación de la MS y de la FDN entre los primeros niveles de fermentación como efecto de la adición de compuestos energéticos, de manera que los valores de DE aumentaron al pasar de una
Degradación, consumo y producción con saccharina Ecosist. Recur. Agropec. 4(12):431-441,2017

dieta sin $\mathrm{MM}(0 \%)$ a una inclusión del $10 \%$, pero se mantuvieron sin cambios significativos en niveles mayores de inclusión, aún en el transcurso del tiempo de fermentación. Es importante resaltar los valores altos de las tasas de degradación, tanto de MS como de FDN, encontrados en la mayoría de las dietas ( $k d M S=0.236, k d F D N=0.254)$, aún cuando la Saccharina es un alimento fermentado con una cinética diferente a otras dietas, donde las bacterias modifican la estructura de los compuestos antes de ser sometidos al proceso de degradación ruminal, lo que cambia las proporciones y la velocidad de degradación del alimento y sus fracciones. Por lo que, en el proceso de preparación de la Saccharina, con la adición de $\mathrm{MM}$, se busca mejorar la calidad y la cantidad del material aprovechable por el animal en el rumen a través del incremento de la fracción soluble y de las kd. En este sentido, el aumento de MM reduce la fracción no degradable de las dietas y aumenta la fracción soluble de la MS, incrementando la DE de todas las dietas superiores al $10 \%$. Aunque en un estudio con Saccharina el valor de kd observado estuvo entre 0.02 a 0.04 (Gaspar-Pinzón et al. 2011). Las diferencias en la kd entre estudios pueden atribuirse a los componentes de la dieta utilizados. Las dietas con valores de kd altos (de Castro-Menezes et al. 2011) se han observado en dietas elaboradas con tallos de caña que tienen mayor cantidad de azúcares y, por consiguiente, mejor degradación, en contraste con la caña integral que arroja menores valores de $\mathrm{kd}$ (López et al. 2003).

A diferencia de los pastos que tienen bajas tasas de degradación y limitaciones físicas (Rosales y Pinzón 2005), en una dieta fermentada, como la Saccharina, la degradación se reduce al mínimo a las $24 \mathrm{~h}$, después de una fase rápida, inicial. Lo que apoya la idea de que, para este alimento fermentado, el proceso de degradación se finaliza antes de las $36 \mathrm{~h}$; y significa, que la fase del alimento que potencialmente se degrada está agotada a esta hora de incubación, y que las incubaciones posteriores no aportarán mucho en términos de la descripción de la cinética del alimento, pero si influyen en el valor de la kd. Una adecuada descripción cinética de los 


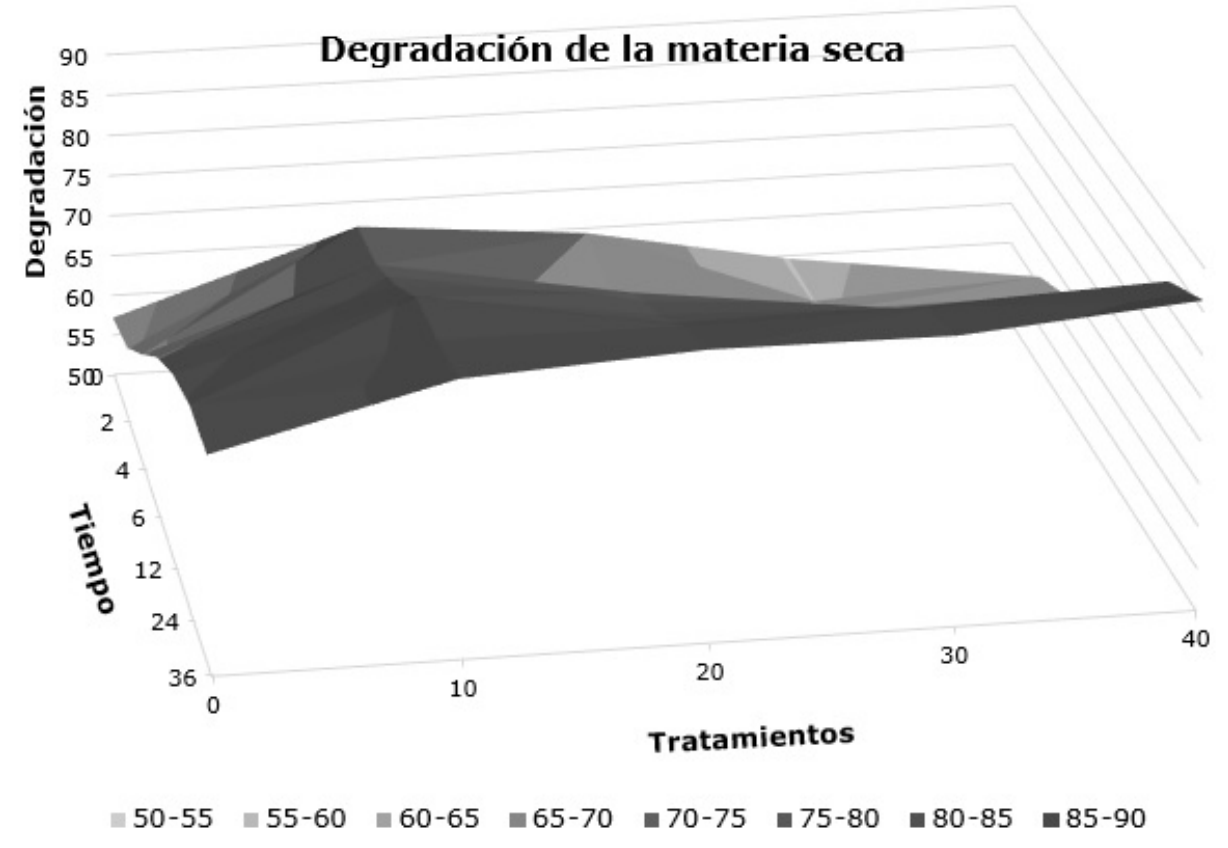

Degradación de la fibra detergente neutra



Figura 1. Degradación in situ de la materia seca (a) y la fibra detergente neutro (b) para todos los tratamientos.

procesos que tienen lugar al inicio de la curva de degradación, donde se da la mayor velocidad, puede mejorar la descripción del proceso biológico, ya que los puntos están distribuidos en el rango de incuba- ción que tiene mayor importancia en la cinética de la degradación ruminal. En este sentido, el tiempo máximo debe estar relacionado con la velocidad del proceso y no a un tiempo predeterminado, que 
Tabla 4. Efecto de la dieta en la fracción no degradable, fracción soluble, tasa de degradación y degradación efectiva de la materia seca y el contenido de fibra detergente neutro (FDN).

\begin{tabular}{lllllllll}
\hline \multirow{2}{*}{ Dieta } & \multicolumn{7}{c}{ Variables } \\
\cline { 2 - 9 } & UMS & FSMS & kdMS & DEMS & UFDN & FSFDN & kdFDN & DEFDN \\
\hline 0 & $24.02^{a}$ & $58.93^{b}$ & $0.236^{a}$ & $73.60^{b}$ & $47.54^{a}$ & $18.87^{c}$ & $0.254^{a}$ & $48.03^{b}$ \\
10 & $14.85^{a b}$ & $71.81^{a}$ & $0.131^{a}$ & $80.86^{a}$ & $22.81^{a b}$ & $48.76^{a}$ & $0.173^{a}$ & $58.89^{a}$ \\
20 & $15.16^{a b}$ & $66.81^{a}$ & $0.089^{a}$ & $79.97^{a}$ & $9.36^{b}$ & $44.39^{a b}$ & $0.065^{a}$ & $57.46^{a}$ \\
30 & $15.76^{a b}$ & $59.84^{b}$ & $0.113^{a}$ & $78.94^{a}$ & $23.61^{a b}$ & $38.29^{a b}$ & $0.070^{a}$ & $50.36^{a b}$ \\
40 & $11.30^{b}$ & $60.50^{b}$ & $0.121^{a}$ & $80.61^{a}$ & $0.001^{b}$ & $35.42^{b}$ & $0.005^{a}$ & $43.81^{b}$ \\
E.E & 3.91 & 1.65 & 0.08 & 1.06 & 17.18 & 3.50 & 0.15 & 2.71 \\
\hline \multicolumn{7}{l}{ abc medias con diferente superíndice en columnas son estadísticamente diferentes $(\mathrm{p} \leq 0.05)}$.
\end{tabular}

Tabla 5. Peso vivo y ganancia de peso de ovinos Pelibuey alimentados con diferentes porcentajes de maíz molido en una dieta integral basada en caña de azúcar fermentada en un periodo de $77 \mathrm{~d}$.

\begin{tabular}{lcccc}
\hline & \multicolumn{4}{c}{ Tratamiento (\% maíz) } \\
\cline { 2 - 5 } & $10 \%$ & $20 \%$ & $30 \%$ & $40 \%$ \\
\hline Peso vivo inicial $(\mathrm{kg})$ & $18.5 \pm 2.6$ & $17.8 \pm 3.7$ & $19.3 \pm 2.3$ & $16.8 \pm 2.1$ \\
Peso vivo final $(\mathrm{kg})$ & $24.5 \pm 4.1$ & $24.8 \pm 5.9$ & $27.5 \pm 2.3$ & $26.16 \pm 3.7$ \\
Peso ganado en el periodo $(\mathrm{kg})$ & $6 \pm 2.4^{a}$ & $7 \pm 3.2^{a}$ & $8.17 \pm 1.9^{a}$ & $9.33 \pm 1.7^{a}$ \\
Ganancia diaria de peso $(\mathrm{kg})$ & $0.08 \pm 0.03^{a}$ & $0.09 \pm 0.04^{a}$ & $0.11 \pm 0.025^{a}$ & $0.12 \pm 0.02^{a}$ \\
Consumo MS total $(\mathrm{kg})$ & $49.32^{c}$ & $51.62^{c}$ & $59.60^{b}$ & $70.69^{a}$ \\
Eficiencia de ganancia de peso & $0.121^{a}$ & $0.136^{a}$ & $0.137^{a}$ & $0.132^{a}$ \\
\hline abc medias con diferente superíndice entre líneas
\end{tabular}

abc medias con diferente superíndice entre líneas son estadísticamente diferentes $(p \leq 0.05)$.

en este caso es cercano a $36 \mathrm{~h}$ de incubación. El aumento en la DE en la dieta al $10 \%$ de MM es una consecuencia de la mayor cantidad de FS en la dieta. Pero el comportamiento similar encontrado al $40 \%$ de inclusión es un reflejo del aumento del material degradable a expensas de la disminución de U. Mientras que la DE obtenida para MS y FDN (Tabla 4) indica que las dietas fermentadas modifican la estructura de las fracciones de las dietas.

Algunos estudios reportan un mejor consumo cuando se incluye entre 10 y $20 \%$ de polvo de arroz en dietas basadas en Saccharina (Ruíz et al. 2005, Waclawovsky et al. 2010). El incremento en el porcentaje de inclusión de MM en la dieta integral de corderos incrementó el peso vivo y mayor ganancia de peso, en el tratamiento con $40 \%$, con el que se obtuvieron $0.12 \mathrm{~kg}$ por día por cordero, pero no se observaron diferencias estadísticas entre las dietas. Lo que se atribuye a la variabilidad entre los animales (Figura 2). Pero al evaluarse la ganancia de peso por medio de contrastes ortogonales se obtuvo diferencias significativas al comparar las dietas de 10 y $20 \%$ vs 30 y $40 \%$ de inclusión, pero no se encontraron diferencias entre la eficiencia de la ganancia de peso.
De acuerdo a los requerimientos de MS de los ovinos indicados por el NRC (2007), sólo la dieta con $40 \%$ de inclusión de MM cubrió la cantidad recomendada, aunque la dieta con $30 \%$, se encontró muy cerca de los niveles de consumo de MS. Otra razón por la cual las ganancias de peso no fueron altas, se pudo deber a que los valores de proteína fueron bajos en todas las dietas y estuvieron en el nivel mínimo recomendado ( $14.5 \%$ de PC) para lograr las ganancias de peso esperadas (NRC 2007), además, parte del nitrógeno provenía de la urea, lo que pudiera explicar la razón de que la dieta con 40 $\%$, a pesar de que cubrió los requerimientos de consumo, no permitió alcanzar las GDP con diferencias significativas respecto a los otros tratamientos, ya que la urea es menos eficiente que otras fuentes de proteína.

Las ganancias de peso obtenidas con la dieta adicionada al $20 \%$ de MM (90 g) es menor a la indicada para hembras (107 g) y machos (150-167 g) por Blardony-Ricardez et al. (2013). Una de las razones pudiera ser el menor porcentaje de proteína cruda en la dieta del presente estudio (14\%) en comparación con el contenido de PC de la dieta con sorgo que tuvo en promedio $20.0 \%$. Una posible 


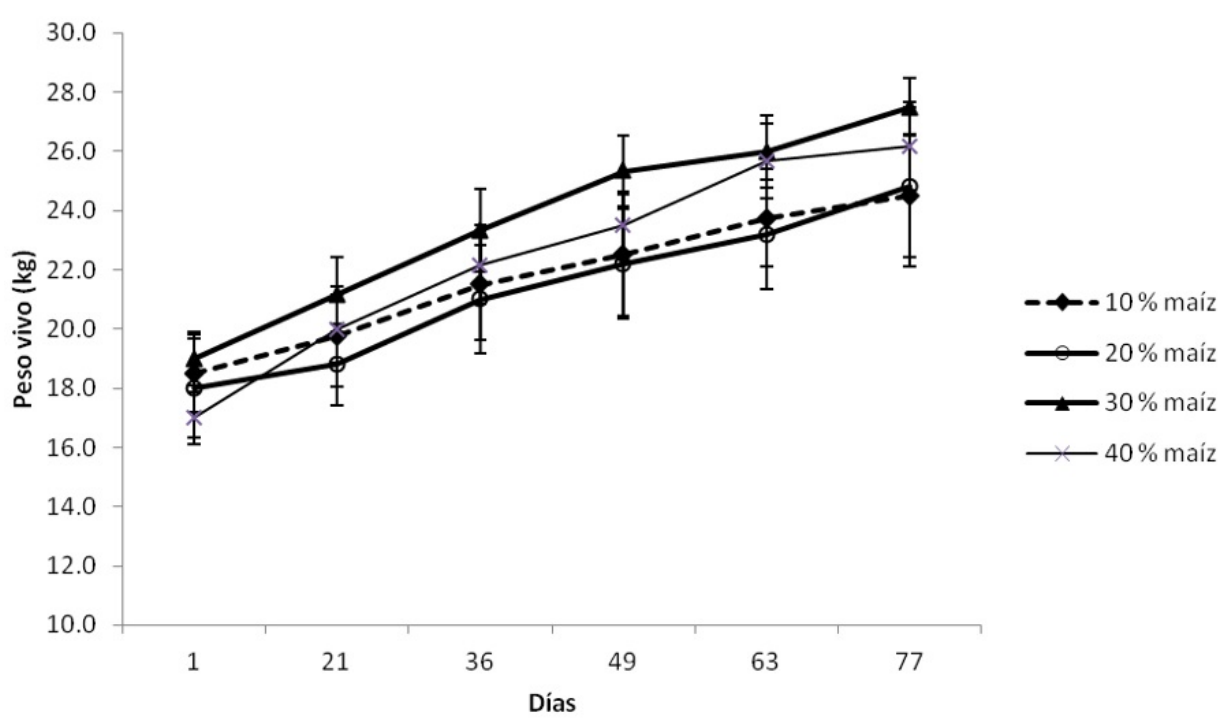

Figura 2. Peso vivo de corderos Pelibuey estabulados y alimentados con Saccharina adicionada con $10,20,30$ o $40 \%$ de maíz molido..

razón de la diferencia en contenido de $\mathrm{PC}$ se atribuye a la calidad del MM, ya que el maíz adquirido de manera comercial para alimento de animales puede incluir algún porcentaje de residuos, mientras que el sorgo generalmente es grano limpio que se utiliza para la alimentación de animales.

\section{CONCLUSIONES}

La adición de maíz molido, a las dietas de Saccharina, mejora la degradación de la MS y FDN desde un nivel de $10 \%$ de inclusión en las dietas. Sin embargo, niveles altos de inclusión no tienen un efecto importante en el aumento de la degradación. El aumento de la degradación efectiva es consecuencia del aumento de la fracción soluble y de la disminución de la fracción no degradable, tanto de la MS como de la FDN, y no de la tasa de degradación. El aumento de nivel de maíz molido en la dieta mejora el consumo voluntario, pero no las ganancias de peso cuando se evalúan individualmente, pero si cuando se contrastan los niveles bajos contra los altos.

\section{AGRADECIMIENTOS}

Al Consejo Nacional de Ciencia y Tecnología (CONACYT) por la beca otorgada a la estudiante Beatriz Godínez Juárez para realizar sus estudios de posgrado en el programa de Producción Agroalimentaria en el Trópico del Colegio de Postgraduados, Campus Tabasco.

\section{LITERATURA CITADA}

Aguilar-Rivera N, Galindo-Mendoza G, Fortanelli- Martínez J, Contreras-Servín C (2011) Factores de competitividad de la agroindustria de la caña de azúcar en México. Región y Sociedad 23: 261-297.

Aguirre J, Magaña R, Martínez S, Gómez A, Ramírez JC, Barajas R, et al. (2010) Caracterización nutricional y uso de la caña de azúcar y residuos transformados en dietas para ovinos. Zootecnia Tropical 28: 489497.

Barahona RB, Sánchez PS (2005) Limitaciones físicas y químicas de la digestibilidad de pastos tropicales y estrategias para aumentarla. Revista Corpoica: Ciencia y Tecnología Agropecuaria 6: 69-82. 
Blardony-Ricardez K, González-Garduño R, Ramos-Juárez J, Díaz-Rivera P, Elías-Iglesias A (2013) Ganancia de peso de corderos alimentados con una dieta integral de Saccharina y un probiótico. Ciencia en la Frontera 11: 29-35.

Cárdenas JR, Aranda EM, Hernández D, Lagunes LDC, Ramos JA, Salgado S (2008) Obtención de un alimento fermentado en estado sólido a partir del bagacillo de retorno, pulido de arroz e inóculos. Su utilización en la alimentación animal. Revista Cubana de Ciencia Agrícola 42: 173-176.

de Castro-Menezes GC, Valadares-Filho SDC, Antunes-Magalhães F, Diniz-Valadares RF, Mariz LD, Detmann $\mathrm{E}$, et al. (2011) Total and partial digestibility, rates of digestion obtained with rumen evacuation and microbial protein synthesis in bovines fed fresh or ensiled sugar cane and corn silage. Revista Brasileria de Zootecnia 40: 1104-1113.

Elías ALO, Lezcano P, Cordero L, Quintana L (1990) Reseña descriptiva sobre el desarrollo de una tecnología de enriquecimiento proteico en la caña de azúcar mediante fermentación en estado sólido (Saccharina). Revista Cubana de Ciencia Agrícola 24: 147-55.

Elías A, Lezcano O (1994). Effect of the inclusion of levels of maize meal on the fermentation of sugar cane. Cuban Journal of Agricultural Science 28: 321-327.

García E (1988) Modificaciones al sistema de clasificación climática de Köppen. $4^{a}$ ed. Instituto de Geografía. Universidad Nacional Autónoma de México. México. 252p.

Gaspar-Pinzón A, Aranda-Ibañez EA, Pérez-Pérez J, Hernández-Garay A, Da Silva-Bueno IC, César-Vitte A (2011) Rumen characteristics of young bulls fed diets based on grass and commercial concentrate supplemented with Hibiscus rosa-sinensis and Saccharin. Ciência Animal Brasileira 12: 26-36.

González-Garduño R, Blardony-Ricardez K, Ramos-Juárez J, Ramírez-Hernández B, Sosa R, Gaona-Ponce M (2013) Rentabilidad de la producción de carne de ovinos Katahdin x Pelibuey con tres tipos de alimentación. Avances en Investigación Agropecuaria 7: 135-148.

Horwitz W, Latimer GW (2005) Official Methods of Analysis of the AOAC International, 18th ed., AOAC International, Gaithersburg, MD, USA. 3000p.

Julián-Ricardo MC, Ramos-Sánchez LB (2007) Fermentación en estado sólido (I). Producción de alimento animal. Tecnología Química 27: 17-22.

López I, Aranda EM, Ramos JA, Mendoza GD (2003) Nutritional evaluation of eight sugarcane varieties with forage potential. Revista Cubana de Ciencia Agrícola 37: 375-380.

Martín PC (2009) El uso de residuales agroindustriales en la alimentación animal en Cuba: pasado, presente y futuro. Avances en Investigación Agropecuaria 13: 3-10.

Missio RL (2016) Treatment of sugarcane bagasse for ruminant feed. Archivos de Zootecnia 65: 267-278.

Mohamed MAA, Babiker IS, Chen ZM, Ikeda K, Ohta K, Kato K (2004) The role of climate variability in the inter-annual variation of terrestrial net primary production (NPP). Science of the Total Environment 332: 123-137.

Monroy JM, Aranda E, Mendoza G, Ramos JA, Herrera J, Cobos M, Izquierdo F (2006) Elaboración y conservación de Saccharina a partir de caña de azúcar integral, con la adición de melaza y pulidura de arroz. Revista Cubana de Ciencia Agrícola 40: 167-172.

Noguera RR, Posada SL (2007) Modelación de la cinética de degradación de alimentos para rumiantes. Revista Colombiana de Ciencia Pecuaria 20: 174-182. 
NRC (2007) Nutrient requirements of small ruminants. Sheep, goats, cervids and new world camelids. National Academy Press. Washington, USA. 347p.

Oliveira D, Souza K, Sampaio LR, da Conceição R, Veloso VJ, Moreira D, et al. (2012) Elephant grass, sugarcane, and rice bran in diets for confined sheep. Tropical Animal Health and Production 44: 18551863.

Olivera A, Aranda IE, Ramos JJ, Vargas VLM, Zaldivar CJ, Mendoza MG (2014) Evaluation of the nutritive value of sugarcane residues inoculated with fungus Fomes sp. Revista MVZ Córdoba 19: 4047-4058.

Orskov ER, McDonald I (1979) The estimate of protein degradability in the rumen from incubation measurements weighted according to rate of passage. Journal of Agricultural Science 92: 499-503.

Ramos J, Elías A, Herrera F (2006) Procesos para la producción de un alimento energético-proteico para animales. Efecto de cuatro fuentes energéticas en la fermentación en estado sólido (FES) de la caña de azúcar. Revista Cubana de Ciencia Agrícola 40: 51-58.

Rosales RB, Pinzón SS (2005) Limitaciones físicas y químicas de la digestibilidad de pastos tropicales y estrategias para aumentarla. Revista Corpoica: Ciencia y Tecnología Agropecuaria 6(1): 69-82.

Ruiz M, Ruiz J, Torres V (2005) Efecto del polvo de arroz en el consumo y la digestibilidad de raciones integrales basadas en Saccharina rústica para ovinos. Revista Cubana de Ciencia Agrícola 39: 575-580.

Salinas-Chavira J, Almaguer LJ, Aguilera-Aceves CE, Zinn RA, Melladoc M, Ruiz-Barrera O (2013) Effect of substitution of sorghum stover with sugarcane top silage on ruminal dry matter degradability of diets and growth performance of feedlot hair lambs. Small Ruminant Research 112: 73-77.

Van Soest PJ, Robertson JB, Lewis BA (1991) Methods for dietary fibre, neutral detergent fibre and nonstarch polysaccharides in relation to animal nutrition. Journal of Dairy Science 74: 3583-3597.

Waclawovsky AJ, Sato PM, Lembke CG, Moore PH, Souza GM (2010) Sugarcane for bioenergy production: an assessment of yield and regulation of sucrose content. Plant Biotechnology Journal 8: 263-276. 
